\title{
Certain Sequence Spaces over the Non-Newtonian Complex Field
}

\author{
Sebiha Tekin and Feyzi Başar \\ Department of Mathematics, Faculty of Arts and Sciences, Fatih University, The Hadimköy Campus, Büyükçekmece, \\ 34500 Istanbul, Turkey \\ Correspondence should be addressed to Feyzi Başar; feyzibasar@gmail.com
}

Received 4 February 2013; Accepted 5 April 2013

Academic Editor: Victor Kovtunenko

Copyright (C) 2013 S. Tekin and F. Başar. This is an open access article distributed under the Creative Commons Attribution License, which permits unrestricted use, distribution, and reproduction in any medium, provided the original work is properly cited.

It is known from functional analysis that in classical calculus, the sets $\omega, \ell_{\infty}, c, c_{0}$ and $\ell_{p}$ of all bounded, convergent, null and $p$ absolutely summable sequences are Banach spaces with their natural norms and they are complete according to the metric reduced from their norm, where $0<p<\infty$. In this study, our main goal is to construct the spaces $\omega^{*}, \ell_{\infty}^{*}, c^{*}, c_{0}^{*}$ and $\ell_{p}^{*}$ over the nonNewtonian complex field $\mathbb{C}^{*}$ and to obtain the corresponding results for these spaces, where $\ddot{0} \ddot{<} p \ddot{<} \infty$.

\section{Preliminaries, Background, and Notations}

A complete ordered field is a system consisting of a set $X$, four binary operations $\dot{+}, \dot{-}, \dot{x}, \dot{j}$ for $X$, and an ordering relation $\dot{<}$ for $X$, all of which behave with respect to the set $X$ exactly as ,,$+- \times, /,<$ behave with respect to the set $\mathbb{R}$ of real numbers. We call $X$ the realm of the complete ordered field, [1, page 32]. A complete ordered field is called arithmetic if its realm is a subset of $\mathbb{R}$. A bijective function with domain $\mathbb{R}$ and range a subset of $\mathbb{R}$ is called a generator. For example, the identity function $I$, exponential function, and the function $x^{3}$ are generators.

Bashirov et al. [2] have recently emphasized on the multiplicative calculus and gave the results with applications corresponding to the well-known properties of derivative and integral in the classical calculus. Quite recently, Uzer [3] has extended the multiplicative calculus to the complex valued functions and gave the statements of some fundamental theorems and concepts of multiplicative complex calculus, and demonstrated some analogies between the multiplicative complex calculus and classical calculus by theoretical and numerical examples. Bashirov and Riza [4] have studied the multiplicative differentiation for complex-valued functions and established the multiplicative Cauchy-Riemann conditions. Bashirov et al. [5] have investigated various problems from different fields which can be modelled more efficiently using multiplicative calculus, in place of Newtonian calculus.
Let $\alpha$ be a generator with range $A$. An arithmetic with range $A$, and operations and ordering relation defined as follows, is called $\alpha$-arithmetic. Let $y, z \in A$. Then, we define the operations $\alpha$-addition $(\dot{+}), \alpha$-subtraction $(\dot{-})$, $\alpha$-multiplication $(\dot{x}), \alpha$-division $(\dot{/})$, and $\alpha$-ordering $(\dot{\leq})$ as follows:

$$
\begin{aligned}
& \alpha \text {-addition : } y \dot{+} z=\alpha\left\{\alpha^{-1}(y)+\alpha^{-1}(z)\right\} \\
& \alpha \text {-subtraction : } y \dot{-} z=\alpha\left\{\alpha^{-1}(y)-\alpha^{-1}(z)\right\} \\
& \alpha \text {-multiplication }: y \dot{\times} z=\alpha\left\{\alpha^{-1}(y) \times \alpha^{-1}(z)\right\} \\
& \alpha \text {-division }(z \neq \dot{0}): y / z=\alpha\left\{\frac{\alpha^{-1}(y)}{\alpha^{-1}(z)}\right\} \\
& \alpha \text {-ordering }: y \leq z \Longleftrightarrow \alpha^{-1}(y) \leq \alpha^{-1}(z) .
\end{aligned}
$$

With the above new operations, $(A, \dot{+}, \dot{-}, \dot{x}, \dot{l}, \dot{\leq})$ is an $\alpha$ arithmetic. In other words, one can easily show that $(A, \dot{+}, \dot{-}, \dot{x}, \dot{l}, \dot{\leq})$ is a complete ordered field. As was seen, $\alpha$ generator generates $\alpha$-arithmetic. For example, the identity function generates classical arithmetic, and exponential function generates geometric arithmetic. Each generator generates exactly one arithmetic and each arithmetic is generated by exactly one generator. We denote $\alpha$-zero by $\dot{0}$ and $\alpha$-one by $\dot{i}$ which are obtained from $\alpha(0)$ and $\alpha(1)$, respectively. $\dot{0}$ and numbers obtained by successive $\alpha$-addition of $\dot{i}$ to $\dot{0}$ with 
numbers obtained by successive $\alpha$-subtraction of $i$ from $\dot{0}$ are called $\alpha$-integers. Thus, $\alpha$-integers are given as follows:

$$
\ldots, \alpha(-2), \alpha(-1), \alpha(0), \alpha(1), \alpha(2), \ldots
$$

Thus, we have for all $n \in \mathbb{Z}$ that $\dot{n}=\alpha(n)$. Let $x \in A$. If $x \dot{0}$ then $x$ is called $\alpha$-positive and if $x \dot{<} \dot{0}$ then $x$ is called $\alpha$-negative. The $\alpha$-absolute value $|x|$ of $x \in A$ is defined by

$$
\dot{|x|} \dot{\mid} \begin{cases}x, & x>\dot{0} \\ \dot{0}, & x=\dot{0} \\ \dot{0}-x, & x<\dot{0}\end{cases}
$$

For any elements $r$ and $s$ in $A$ with $r \dot{<} s$, the set of all elements $x$ in $A$ such that $r \dot{\leq} x \dot{\leq} s$ is called an $\alpha$-interval, is denoted by $\dot{[} r, s]$, has $\alpha$-extent of $s \dot{-} r$, and has the $\alpha$-interior consisting of all elements $x$ in $A$ such that $r \dot{<} x \dot{<} s$. Let $\left(u_{n}\right)$ be an infinite sequence of the elements in $A$. Then there is at most one element $u$ in $A$ such that every $\alpha$-interval with $u$ in its $\alpha$-interior contains all but finitely many terms of $\left(u_{n}\right)$. If there is such a number $u$, then $\left(u_{n}\right)$ is said to be $\alpha$-convergent to $u$, which is called the $\alpha$-limit of $\left(u_{n}\right)$. In other words,

$$
\begin{aligned}
& \lim _{n \rightarrow \infty} u_{n}=u(\alpha \text {-convergent }) \\
& \Longleftrightarrow \forall \varepsilon \dot{>} \dot{0}, \exists n_{0} \in \mathbb{N} \ni\left|u_{n} \dot{-} u\right| \dot{<} \varepsilon \\
& \forall n \geq n_{0} \text { and some } u \in A .
\end{aligned}
$$

Let $\alpha$ and $\beta$ be two arbitrarily selected generators and let $*$-("star") also be the ordered pair of arithmetics $(\alpha$ arithmetic, $\beta$-arithmetic). $(B, \ddot{+}, \ddot{-}, \ddot{x}, \ddot{j}, \ddot{\leq})$ is a $\beta$-arithmetic. Definitions given for $\alpha$-arithmetic are also valid for $\beta$-arithmetic. For example, $\beta$-convergence is defined by means of $\beta$-intervals and their $\beta$-interiors.

In the $*$-calculus, $\alpha$-arithmetic is used for arguments and $\beta$-arithmetic is used for values; in particular, changes in arguments and values are measured by $\alpha$-differences and $\beta$-differences, respectively. The operators of the $*$-calculus are applied only to functions with arguments in $A$ and values in $B$. The *-limit of a function $f$ at an element $a$ in $A$ is, if it exists, the unique number $b$ in $B$ such that for every sequence $\left(a_{n}\right)$ of arguments of $f$ distinct from $a$, if $\left(a_{n}\right)$ is $\alpha$-convergent to $a$, then $\left\{f\left(a_{n}\right)\right\} \beta$-converges to $b$ and is denoted by $\lim _{x \rightarrow a}^{*} f(x)=b$. That is,

$$
\begin{array}{r}
\lim _{x \rightarrow a}^{*} f(x)=b \Longleftrightarrow \forall \epsilon \ddot{>} \ddot{0}, \exists \delta \dot{>0} \ni \ddot{\mid} f(x) \ddot{-} b \mid \ddot{<} \epsilon \\
\forall x \in A \text { with }|x \dot{-} a| \dot{<} \delta .
\end{array}
$$

A function $f$ is $*$-continuous at a point $a$ in $A$ if and only if $a$ is an argument of $f$ and $\lim _{x \rightarrow a}^{*} f(x)=f(a)$. When $\alpha$ and $\beta$ are the identity function $I$, the concepts of $*$-limit and $*$-continuity are identical with those of classical limit and classical continuity.

The isomorphism from $\alpha$-arithmetic to $\beta$-arithmetic is the unique function $\iota$ (iota) that possesses the following three properties: (i) $\iota$ is one to one.

(ii) $\iota$ is from $A$ onto $B$.

(iii) For any numbers $u$ and $v$ in $A$,

$$
\begin{aligned}
& \iota(u \dot{+} v)=\iota(u) \ddot{+} \iota(v) \\
& \iota(u \dot{-} v)=\iota(u) \ddot{-} \iota(v) \\
& \iota(u \dot{\times} v)=\iota(u) \ddot{\times} \iota(v) \\
& \iota(u \dot{j} v)=\iota(u) \ddot{l} \iota(v), \quad v \neq \dot{0} \\
& u \dot{\leq} v \Longleftrightarrow \iota(u) \ddot{\leq} \iota(v) .
\end{aligned}
$$

It turns out that $\iota(x)=\beta\left\{\alpha^{-1}(x)\right\}$ for every $x$ in $A$, and that $\iota(\dot{n})=\ddot{n}$ for every integer $n$. Since, for example, $u \dot{+} v$ $=\iota^{-1}\{\iota(u) \ddot{+} \iota(v)\}$, it should be clear that any statement in $\alpha$-arithmetic can readily be transformed into a statement in $\beta$-arithmetic.

\section{Non-Newtonian Complex Field and Some Inequalities}

Let $\dot{a} \in(A, \dot{+}, \dot{-}, \dot{x}, \dot{l}, \dot{\leq})$ and $\ddot{b} \in(B, \ddot{+}, \ddot{-}, \ddot{x}, \ddot{l}, \ddot{\leq})$ be arbitrarily chosen elements from corresponding arithmetics. Then the ordered pair $(\dot{a}, \ddot{b})$ is called as a $*$-point. The set of all $*$-points is called the set of non-Newtonian complex numbers and is denoted by $\mathbb{C}^{*}$; that is,

$$
\begin{aligned}
\mathbb{C}^{*} & :=\left\{z^{*}=\dot{a} \oplus\left(i^{*} \odot \dot{b}\right): a, b \in \mathbb{R}, i^{*}=(\dot{0}, \ddot{1})\right\} \\
& =\left\{z^{*}=(\dot{a}, \ddot{b}): \dot{a} \in A \subseteq \mathbb{R}, \ddot{b} \in B \subseteq \mathbb{R}\right\} .
\end{aligned}
$$

The binary operations addition $(\oplus)$ and multiplication $(\odot)$ of non-Newtonian complex numbers $z_{1}^{*}=\left(\dot{a}_{1}, \ddot{b}_{1}\right)$ and $z_{2}^{*}=$ $\left(\dot{a}_{2}, \ddot{b}_{2}\right)$ are defined, as follows:

$$
\begin{array}{r}
\oplus: \mathbb{C}^{*} \times \mathbb{C}^{*} \longrightarrow \mathbb{C}^{*} \\
\left(z_{1}^{*}, z_{2}^{*}\right) \longmapsto z_{1}^{*} \oplus z_{2}^{*}=\left(\dot{a}_{1} \dot{+} \dot{a}_{2}, \ddot{b}_{1} \ddot{+} \ddot{b}_{2}\right), \\
\odot: \mathbb{C}^{*} \times \mathbb{C}^{*} \longrightarrow \mathbb{C}^{*} \\
\left(z_{1}^{*}, z_{2}^{*}\right) \longmapsto z_{1}^{*} \odot z_{2}^{*}=\left(\alpha\left(\overline{\dot{a}}_{1} \overline{\dot{a}}_{2}-\overline{\ddot{b}}_{1} \overline{\ddot{b}}_{2}\right),\right. \\
\left.\beta\left(\overline{\dot{a}}_{1} \overline{\ddot{b}}_{2}+\overline{\ddot{b}}_{1} \overline{\dot{a}}_{2}\right)\right),
\end{array}
$$

where $\dot{a}_{1}, \dot{a}_{2} \in A$ and $\ddot{b}_{1}, \ddot{b}_{2} \in B$ with

$$
\begin{aligned}
& \overline{\dot{a}}_{1}=\alpha^{-1}\left(\dot{a}_{1}\right)=\alpha^{-1}\left(\alpha\left(a_{1}\right)\right)=a_{1} \in \mathbb{R}, \\
& \bar{b}_{1}=\beta^{-1}\left(\ddot{b}_{1}\right)=\beta^{-1}\left(\beta\left(b_{1}\right)\right)=b_{1} \in \mathbb{R} .
\end{aligned}
$$

Then we have the following.

Theorem 1. $\left(\mathbb{C}^{*}, \oplus, \odot\right)$ is a field.

Proof. A straightforward calculation leads to the following statements: 
(i) $\left(\mathbb{C}^{*}, \oplus\right)$ is an Abelian group;

(ii) $\left(\mathbb{C}^{*} \backslash\left\{\theta^{*}\right\}, \odot\right)$ is an Abelian group;

(iii) the operation $\odot$ is distributive over the operation $\oplus$

which conclude that $\left(\mathbb{C}^{*}, \oplus, \odot\right)$ is a field.

Let $\ddot{b} \in B \subseteq \mathbb{R}$. Then the number $\ddot{b} \ddot{x} \ddot{b}$ is called the $\beta$ square of $\ddot{b}$ and is denoted by $b^{2}$. Let $\ddot{b}$ be a non-negative number in $B$. Then, $\beta\left[\sqrt{\beta^{-1}(\ddot{b})}\right]$ is called the $\beta$-square root of $\ddot{b}$ and is denoted by $\sqrt{\ddot{b}}$. The $*$-distance $d^{*}$ between two arbitrarily elements $z_{1}^{*}=\left(\dot{a}_{1}, \ddot{b}_{1}\right)$ and $z_{2}^{*}=\left(\dot{a}_{2}, \ddot{b}_{2}\right)$ of the set $\mathbb{C}^{*}$ is defined by

$$
\begin{aligned}
d^{*}: \mathbb{C}^{*} \times \mathbb{C}^{*} & \longrightarrow\left[\ddot{0}, \infty \ddot{)}=B^{\prime} \subset B\right. \\
\left(z_{1}^{*}, z_{2}^{*}\right) \longrightarrow d^{*}\left(z_{1}^{*}, z_{2}^{*}\right) & =\sqrt{\left[\iota\left(\dot{a}_{1} \dot{-} \dot{a}_{2}\right)\right]^{\ddot{2}} \ddot{+}\left(\ddot{b}_{1} \ddot{-} \ddot{b}_{2}\right)^{\ddot{2}}} \\
& =\beta\left[\sqrt{\left(a_{1}-a_{2}\right)^{2}+\left(b_{1}-b_{2}\right)^{2}}\right] .
\end{aligned}
$$

Up to now, we know that $\mathbb{C}^{*}$ is a field and the distance between two points in $\mathbb{C}^{*}$ is computed by the function $d^{*}$, defined by (10). Now, we define the $*$-norm and next derive some required inequalities in the sense of non-Newtonian complex calculus.

Let $z^{*} \in \mathbb{C}^{*}$ be an arbitrary element. $d^{*}\left(z^{*}, \theta^{*}\right)$ is called *-norm of $z^{*}$ and is denoted by $\ddot{\|} \cdot \ddot{i}$. In other words,

$$
\begin{aligned}
\ddot{\|} z^{*} \ddot{\|} & =d^{*}\left(z^{*}, \theta^{*}\right) \\
& =\sqrt{[\iota(\dot{a}-\dot{0})]^{\ddot{2}} \ddot{+}(\ddot{b} \ddot{-} \ddot{0})^{\ddot{2}}} \\
& =\beta\left(\sqrt{a^{2}+b^{2}}\right),
\end{aligned}
$$

where $z^{*}=(\dot{a}, \ddot{b})$ and $\theta^{*}=(\dot{0}, \ddot{0})$. Moreover, since for all $z_{1}^{*}, z_{2}^{*} \in \mathbb{C}^{*}$ we have $d^{*}\left(z_{1}^{*}, z_{2}^{*}\right)=\ddot{\|} z_{1}^{*} \ominus z_{2}^{*} \ddot{\|}, d^{*}$ is the induced metric from the norm $\ddot{\|} \cdot \dot{\|}$.

Lemma 2 (*-Triangle inequality). Let $z_{1}^{*}, z_{2}^{*} \in \mathbb{C}^{*}$. Then,

$$
\ddot{\|} z_{1}^{*} \oplus z_{2}^{*} \ddot{\|} \ddot{\leq} \ddot{\|} z_{1}^{*} \ddot{\|} \ddot{+} \ddot{\|} z_{2}^{*} \ddot{\|} .
$$

Proof. Let $z_{1}^{*}, z_{2}^{*} \in \mathbb{C}^{*}$. Then, a straightforward calculation gives that

$$
\begin{aligned}
\ddot{\|} z_{1}^{*} \oplus z_{2}^{*} \ddot{\|} \\
\quad=\sqrt{\left[\iota\left(\dot{a}_{1}+\dot{a}_{2}\right)\right]^{\ddot{+}} \ddot{+}\left(\ddot{b}_{1} \ddot{+} \ddot{b}_{2}\right)^{2}} \\
\quad=\beta\left[\sqrt{\left(a_{1}+a_{2}\right)^{2}+\left(b_{1}+b_{2}\right)^{2}}\right] \\
\quad \ddot{\leq} \beta\left(\sqrt{a_{1}^{2}+b_{1}^{2}}+\sqrt{a_{2}^{2}+b_{2}^{2}}\right)
\end{aligned}
$$

$$
\begin{aligned}
& =\beta\left\{\beta^{-1}\left[\beta\left(\sqrt{a_{1}^{2}+b_{1}^{2}}\right)\right]+\beta^{-1}\left[\beta\left(\sqrt{a_{2}^{2}+b_{2}^{2}}\right)\right]\right\} \\
& =\beta\left[\beta^{-1}\left(\ddot{\|} z_{1}^{*} \ddot{i}\right)+\beta^{-1}\left(\ddot{\|} z_{2}^{*} \ddot{i}\right)\right] \\
& =\ddot{\|} z_{1}^{*} \ddot{\|} \ddot{+} \ddot{\|} z_{2}^{*} \ddot{\|} \text {. }
\end{aligned}
$$

Hence, the inequality (12) holds.

Lemma 3. $\ddot{\|} z_{1}^{*} \odot z_{2}^{*} \ddot{\|}=\ddot{\|} z_{1}^{*} \ddot{\|} \ddot{x} \ddot{\|} z_{2}^{*} \ddot{\|}$ for all $z_{1}^{*}, z_{2}^{*} \in \mathbb{C}^{*}$.

Proof. Let $z_{1}^{*}, z_{2}^{*} \in \mathbb{C}^{*}$. In this case, one can observe by a routine verification that

$$
\begin{aligned}
& \ddot{\|} z_{1}^{*} \odot z_{2}^{*} \ddot{\|} \\
& =\sqrt{\left\{\iota\left[\alpha\left(\overline{\dot{a}}_{1} \overline{\dot{a}}_{2}-\overline{\vec{b}}_{1} \overline{\vec{b}}_{2}\right)\right]\right\}^{\ddot{2}} \ddot{+}\left[\beta\left(\overline{\dot{a}}_{1} \overline{\vec{b}}_{2}+\overline{\vec{b}}_{1} \overline{\dot{a}}_{2}\right)\right]^{\ddot{2}}} \\
& =\beta\left[\sqrt{\left(a_{1} a_{2}-b_{1} b_{2}\right)^{2}+\left(a_{1} b_{2}+b_{1} a_{2}\right)^{2}}\right] \\
& =\beta\left(\sqrt{a_{1}^{2}+b_{1}^{2}} \sqrt{a_{2}^{2}+b_{2}^{2}}\right) \\
& =\beta\left\{\beta^{-1}\left[\beta\left(\sqrt{a_{1}^{2}+b_{1}^{2}}\right)\right] \times \beta^{-1}\left[\beta\left(\sqrt{a_{2}^{2}+b_{2}^{2}}\right)\right]\right\} \\
& =\beta\left[\beta^{-1}\left(\ddot{\|} z_{1}^{*} \ddot{i}\right) \times \beta^{-1}\left(\ddot{\|} z_{2}^{*} i \ddot{i}\right)\right] \\
& =\ddot{\|} z_{1}^{*} \ddot{\|} \ddot{x} \ddot{\|} z_{2}^{*} \ddot{\|} \text {, }
\end{aligned}
$$

as required.

Lemma 4. Let $z_{1}^{*}, z_{2}^{*} \in \mathbb{C}^{*}$. Then, the following inequality holds:

$$
\begin{aligned}
& \ddot{\|} z_{1}^{*} \oplus z_{2}^{*} \ddot{\|} \ddot{j}\left(\ddot{i} \ddot{+} \ddot{\|} z_{1}^{*} \oplus z_{2}^{*} \ddot{i}\right) \\
& \ddot{\leq} \ddot{\|} z_{1}^{*} \ddot{\|} \ddot{l}\left(\ddot{1} \ddot{+} \ddot{\|} z_{1}^{*} \ddot{i}\right) \ddot{+} \ddot{\|} z_{2}^{*} \ddot{\|} \ddot{j}\left(\ddot{1} \ddot{+} \ddot{\|} z_{2}^{*} \ddot{\|}\right) .
\end{aligned}
$$

Proof. Let $z_{1}^{*}, z_{2}^{*} \in \mathbb{C}^{*}$. Then, one can see that

$$
\begin{aligned}
& \ddot{\|} z_{1}^{*} \oplus z_{2}^{*} \ddot{\|} \ddot{l}\left(\ddot{i} \ddot{+} \ddot{\|} z_{1}^{*} \oplus z_{2}^{*} \ddot{i}\right) \\
& =\beta\left[\sqrt{\left(a_{1}+a_{2}\right)^{2}+\left(b_{1}+b_{2}\right)^{2}}\right] \\
& \ddot{j}\left\{\ddot{1} \ddot{+} \beta\left[\sqrt{\left(a_{1}+a_{2}\right)^{2}+\left(b_{1}+b_{2}\right)^{2}}\right]\right\} \\
& =\beta\left[\frac{\sqrt{\left(a_{1}+a_{2}\right)^{2}+\left(b_{1}+b_{2}\right)^{2}}}{1+\sqrt{\left(a_{1}+a_{2}\right)^{2}+\left(b_{1}+b_{2}\right)^{2}}}\right] \\
& \ddot{\leq} \beta\left[\left(\frac{\sqrt{a_{1}^{2}+b_{1}^{2}}}{1+\sqrt{a_{1}^{2}+b_{1}^{2}}}\right)+\left(\frac{\sqrt{a_{2}^{2}+b_{2}^{2}}}{1+\sqrt{a_{2}^{2}+b_{2}^{2}}}\right)\right]
\end{aligned}
$$




$$
\begin{aligned}
& =\beta\left\{\frac{\beta^{-1}\left[\beta\left(\sqrt{a_{1}^{2}+b_{1}^{2}}\right)\right]}{\beta^{-1}\left\{\beta\left[\beta^{-1}(\ddot{1})+\beta^{-1}\left[\beta\left(\sqrt{a_{1}^{2}+b_{1}^{2}}\right)\right]\right]\right\}}\right. \\
& \left.+\frac{\beta^{-1}\left[\beta\left(\sqrt{a_{2}^{2}+b_{2}^{2}}\right)\right]}{\beta^{-1}\left\{\beta\left\{\beta^{-1}(\ddot{1})+\beta^{-1}\left[\beta\left(\sqrt{a_{2}^{2}+b_{2}^{2}}\right)\right]\right\}\right\}}\right\} \\
& =\beta\left\{\left[\frac{\beta^{-1}\left(\ddot{\|} z_{1}^{*} \ddot{\|}\right)}{\beta^{-1}\left(\ddot{1} \ddot{+} \ddot{\|} z_{1}^{*} \ddot{i}\right)}\right]+\left[\frac{\beta^{-1}\left(\ddot{\|} z_{2}^{*} \ddot{\|}\right)}{\beta^{-1}\left(\ddot{i} \ddot{+} \ddot{\|} z_{2}^{*} \ddot{\|}\right)}\right]\right\} \\
& =\beta\left\{\beta^{-1}\left\{\beta\left[\frac{\beta^{-1}\left(\ddot{\|} z_{1}^{*} \ddot{\|}\right)}{\beta^{-1}\left(\ddot{i} \ddot{+} \ddot{\|} z_{1}^{*} \ddot{i}\right)}\right]\right\}\right. \\
& \left.+\beta^{-1}\left\{\beta\left[\frac{\beta^{-1}\left(\ddot{\|} z_{2}^{*} \ddot{\|}\right)}{\beta^{-1}\left(\ddot{1} \ddot{+} \ddot{\|} z_{2}^{*} \ddot{\|}\right)}\right]\right\}\right\} \\
& =\beta\left\{\beta^{-1}\left[\ddot{\|} z_{1}^{*} \ddot{\|} \ddot{j}\left(\ddot{1} \ddot{+} \ddot{\|} z_{1}^{*} \ddot{i}\right)\right]+\beta^{-1}\left[\ddot{\|} z_{2}^{*} \ddot{\|} \ddot{j}\left(\ddot{1} \ddot{+} \ddot{\|} z_{2}^{*} \ddot{i}\right)\right]\right\} \\
& =\ddot{\|} z_{1}^{*} \ddot{\|} \ddot{j}\left(\ddot{1} \ddot{+} \ddot{\|} z_{1}^{*} \ddot{\|}\right) \ddot{+} \ddot{\|} z_{2}^{*} \ddot{\|} \ddot{j}\left(\ddot{1} \ddot{+} \ddot{\|} z_{2}^{*} \ddot{\|}\right) \text {. }
\end{aligned}
$$

This means that the inequality (15) holds.

Lemma 5 (*-Minkowski inequality). Let $p \ddot{\geq} \ddot{1}$ and $z_{k}^{*}, t_{k}^{*} \in$ $\mathbb{C}^{*}$ for all $k \in\{1,2,3, \ldots, n\}$. Then,

$$
\left(\sum_{k=0}^{n} \ddot{\|} z_{k}^{*} \oplus t_{k}^{*} \ddot{\|}^{p}\right)^{1 / p} \ddot{\leq}\left(\sum_{k=0}^{n} \ddot{\|} z_{k}^{*} \ddot{\|}^{p}\right)^{1 / p} \ddot{+}\left(\sum_{k=0}^{n} \ddot{\|} t_{k}^{*} \ddot{\|}^{p}\right)^{1 / p} .
$$

Proof. Let $p \ddot{\geq} \ddot{1}$ and $z_{k}^{*}, t_{k}^{*} \in \mathbb{C}^{*}$ for all $k \in\{1,2,3, \ldots, n\}$. Then,

$$
\begin{aligned}
\sum_{k=0}^{n} \ddot{\|} z_{k}^{*} \oplus t_{k}^{*} \ddot{\|}^{p} & \\
= & \ddot{\|} z_{0}^{*} \oplus t_{0}^{*} \ddot{\|^{p}} \ddot{+} \ddot{\|} z_{1}^{*} \oplus t_{1}^{*} \ddot{\|}^{p} \ddot{+} \cdots \ddot{+} \ddot{\|} z_{n}^{*} \oplus t_{n}^{*} \ddot{\|}^{p} \\
=\beta[ & \beta^{-1}\left(\ddot{\|} z_{0}^{*} \oplus t_{0}^{*} \ddot{\|}^{p}\right)+\beta^{-1}\left(\ddot{\|} z_{1}^{*} \oplus t_{1}^{*} \ddot{\|}^{p}\right) \\
& \left.+\cdots+\beta^{-1}\left(\ddot{\|} z_{n}^{*} \oplus t_{n}^{*} \ddot{\|}^{p}\right)\right] .
\end{aligned}
$$

Let us take $z_{k}^{*}=\left(\dot{x}_{k}, \ddot{y}_{k}\right), z_{k}=\left(x_{k}, y_{k}\right), t_{k}^{*}=\left(\dot{u}_{k}, \ddot{v}_{k}\right), t_{k}=$ $\left(u_{k}, v_{k}\right)$. Then, since the equality

$$
\begin{aligned}
\ddot{\|} z_{k}^{*} \oplus t_{k}^{*} \ddot{\|} \\
\quad=\sqrt{\left[\iota\left(\dot{x}_{k}+\dot{u}_{k}\right)\right]^{\ddot{2}} \ddot{+}\left(\ddot{y}_{k} \ddot{+} \ddot{v}_{k}\right)^{\ddot{2}}} \\
\quad=\beta\left[\sqrt{\left(x_{k}+u_{k}\right)^{2}+\left(y_{k}+v_{k}\right)^{2}}\right] \\
=\beta\left(\left|z_{k}+t_{k}\right|\right)
\end{aligned}
$$

holds for every fixed $k$, we obtain

$$
\begin{aligned}
& \ddot{\|} z_{k}^{*} \oplus t_{k}^{*} \ddot{i}^{p}
\end{aligned}
$$

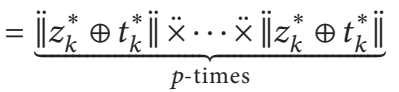

$$
\begin{aligned}
& =\beta\left[\beta^{-1}\left(\ddot{\|} z_{k}^{*} \oplus t_{k}^{*} \ddot{i}\right) \times \cdots \times \beta^{-1}\left(\ddot{\|} z_{k}^{*} \oplus t_{k}^{*} \ddot{i}\right)\right] \\
& =\beta\left\{\beta^{-1}\left[\beta\left(\left|z_{k}+t_{k}\right|\right)\right] \times \cdots \times \beta^{-1}\left[\beta\left(\left|z_{k}+t_{k}\right|\right)\right]\right\} \\
& =\beta\left(\left|z_{k}+t_{k}\right|^{\beta^{-1}(p)}\right)
\end{aligned}
$$

by (18) and Minkowski inequality in the complex field leads us to

$$
\begin{aligned}
& \sum_{k=0}^{n} \ddot{\|} z_{k}^{*} \oplus t_{k}^{*} \ddot{\|}^{p} \\
&=\beta\left\{\beta^{-1}\left[\beta\left(\left|z_{0} \oplus t_{0}\right|^{\beta^{-1}(p)}\right)\right]+\beta^{-1}\left[\beta\left(\left|z_{1} \oplus t_{1}\right|^{\beta^{-1}(p)}\right)\right]\right. \\
&\left.\quad+\cdots+\beta^{-1}\left[\beta\left(\left|z_{n} \oplus t_{n}\right|^{\beta^{-1}(p)}\right)\right]\right\} \\
&=\beta\left(\sum_{k=0}^{n}\left|z_{k}+t_{k}\right|^{\beta^{-1}(p)}\right) \\
& \ddot{\leq} \beta\left\{\left[\left(\sum_{k=0}^{n}\left|z_{k}\right|^{\beta^{-1}(p)}\right)^{1 / \beta^{-1}(p)}\right.\right. \\
&\left.\left.\quad+\left(\sum_{k=0}^{n}\left|t_{k}\right|^{\beta^{-1}(p)}\right)^{1 / \beta^{-1}(p)}\right]^{\beta^{-1}(p)}\right\},
\end{aligned}
$$

$$
\begin{aligned}
& \beta^{-1}\left\{\left[\left(\sum_{k=0}^{n} \ddot{\|} z_{k}^{*} \ddot{\|}^{p}\right)^{1 \ddot{p}} \ddot{+}\left(\sum_{k=0}^{n} \ddot{\|} t_{k}^{*} \ddot{\|}^{p}\right)^{1 / p}\right]^{p}\right\}
\end{aligned}
$$

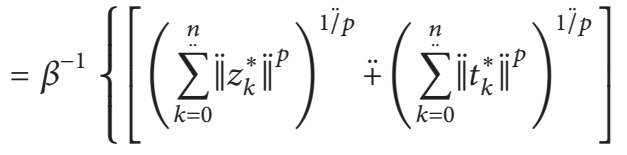

$$
\begin{aligned}
& \left.\ddot{\times} \cdots \ddot{x}\left[\left(\sum_{k=0}^{n} \ddot{\|} z_{k}^{*} \ddot{\|}^{p}\right)^{1 / p} \ddot{+}\left(\sum_{k=0}^{n} \ddot{\|} t_{k}^{*} \ddot{\|}^{p}\right)^{1 / p}\right]\right\} \\
& =\beta^{-1}\left[\left(\sum_{k=0}^{n} \ddot{\|} z_{k}^{*} \ddot{\|}^{p}\right)^{1 / p} \ddot{+}\left(\sum_{k=0}^{n} \ddot{\|} t_{k}^{*} \ddot{\|}^{p}\right)^{1 / p}\right] \\
& \times \cdots \times \beta^{-1}\left[\left(\sum_{k=0}^{n} \ddot{\|} z_{k}^{*} \ddot{\|}^{p}\right)^{1 / p} \ddot{+}\left(\sum_{k=0}^{n} \ddot{\|} t_{k}^{*} \ddot{\|}^{p}\right)^{1 / p}\right]
\end{aligned}
$$




$$
\begin{aligned}
& =\left\{\beta^{-1}\left[\left(\sum_{k=0}^{n} \ddot{\|} z_{k}^{*} \ddot{\|}^{p}\right)^{1 / p}\right]+\beta^{-1}\left[\left(\sum_{k=0}^{n} \ddot{\|} t_{k}^{*} \ddot{\|}^{p}\right)^{1 / p}\right]\right\} \\
& \times \cdots \times\left\{\beta^{-1}\left[\left(\sum_{k=0}^{n} \ddot{\|} z_{k}^{*} \ddot{\|}^{p}\right)^{1 / p}\right]\right. \\
& \left.+\beta^{-1}\left[\left(\sum_{k=0}^{n} \ddot{\|} t_{k}^{*} \ddot{\|}^{p}\right)^{1 / p}\right]\right\} \\
& =\left\{\beta^{-1}\left[\left(\sum_{k=0}^{n} \ddot{\|} z_{k}^{*} \ddot{\|}^{p}\right)^{1 / p}\right]+\beta^{-1}\left[\left(\sum_{k=0}^{n} \ddot{\|} t_{k}^{*} \ddot{\|}^{p}\right)^{1 / p}\right]\right\}^{\beta^{-1}(p)} .
\end{aligned}
$$

On the other hand, let us prove

$$
\beta\left[\left(\sum_{k=0}^{n}\left|z_{k}\right|^{\beta^{-1}(p)}\right)^{1 / \beta^{-1}(p)}\right]=\left(\sum_{k=0}^{n} \ddot{\|} z_{k}^{*} \ddot{i}^{p}\right)^{1 / p} .
$$

Indeed,

$$
\begin{aligned}
& \left\{\beta\left[\left(\sum_{k=0}^{n}\left|z_{k}\right|^{\beta^{-1}(p)}\right)^{1 / \beta^{-1}(p)}\right]\right\}^{p} \\
& =\underbrace{\beta\left[\left(\sum_{k=0}^{n}\left|z_{k}\right|^{\beta^{-1}(p)}\right)^{1 / \beta^{-1}(p)}\right] \ddot{x} \cdots \ddot{x} \beta\left[\left(\sum_{k=0}^{n}\left|z_{k}\right|^{\beta^{-1}(p)}\right)^{1 / \beta^{-1}(p)}\right]}_{p \text {-times }} \\
& =\beta\left[\left(\sum_{k=0}^{n}\left|z_{k}\right|^{\beta^{-1}(p)}\right)^{1 / \beta^{-1}(p)}\right. \\
& \left.\quad \times \cdots \times\left(\sum_{k=0}^{n}\left|z_{k}\right|^{\beta^{-1}(p)}\right)^{1 / \beta^{-1}(p)}\right] \\
& =\beta\left\{\left[\left(\sum_{k=0}^{n}\left|z_{k}\right|^{\beta^{-1}(p)}\right)^{1 / \beta^{-1}(p)}\right]^{\beta^{-1}(p)}\right\} \\
& =\beta\left(\sum_{k=0}^{n}\left|z_{k}\right|^{\beta^{-1}(p)}\right) \\
& =\sum_{k=0}^{n} \ddot{\|} z_{k}^{*} \ddot{H}^{p} .
\end{aligned}
$$

Substituting the relation (24) in (22) we obtain,

$$
\begin{aligned}
\beta^{-1}\left\{\left[\left(\sum_{k=0}^{n} \ddot{\|} z_{k}^{*} \ddot{\|}^{p}\right)^{1 / p} \ddot{+}\left(\sum_{k=0}^{n} \ddot{\|} t_{k}^{*} \ddot{i}^{p}\right)^{1 / p}\right]^{p}\right\} \\
=\left[\left(\sum_{k=0}^{n}\left|z_{k}\right|^{\beta^{-1}(p)}\right)^{1 / \beta^{-1}(p)}+\left(\sum_{k=0}^{n}\left|t_{k}\right|^{\beta^{-1}(p)}\right)^{1 / \beta^{-1}(p)}\right]^{\beta^{-1}(p)} .
\end{aligned}
$$

By using this equality in (21), we get

$$
\begin{aligned}
& \left(\sum_{k=0}^{n} \ddot{\|} z_{k}^{*} \oplus t_{k}^{*} \ddot{\|}^{p}\right)^{1 / p} \\
& \ddot{\leq}\left(\sum_{k=0}^{n} \ddot{\|} z_{k}^{*} \ddot{\|}^{p}\right)^{1 / p} \ddot{+}\left(\sum_{k=0}^{n} \ddot{\|} t_{k}^{*} \ddot{\|}^{p}\right)^{1 / p},
\end{aligned}
$$

as desired.

Theorem 6. $\left(\mathbb{C}^{*}, d^{*}\right)$ is a complete metric space, where $d^{*}$ is defined by (10).

Proof. First, we show that $d^{*}$, defined by (10), is a metric on $\mathbb{C}^{*}$.

It is immediate for $z_{1}^{*}, z_{2}^{*} \in \mathbb{C}^{*}$ that

$$
\begin{aligned}
d^{*}\left(z_{1}^{*}, z_{2}^{*}\right) & =\sqrt{\left[\iota\left(\dot{a}_{1}-\dot{a}_{2}\right)\right]^{\ddot{2}} \ddot{+}\left(\ddot{b}_{1} \ddot{-} \ddot{b}_{2}\right)^{\ddot{2}}} \\
& =\beta\left[\sqrt{\left(a_{1}-a_{2}\right)^{2}+\left(b_{1}-b_{2}\right)^{2}}\right] \ddot{\geq} \ddot{0} .
\end{aligned}
$$

(i) Now we show that $d^{*}\left(z_{1}^{*}, z_{2}^{*}\right)=\ddot{0}$ if and only if $z_{1}^{*}=$ $z_{2}^{*}$ for $z_{1}^{*}, z_{2}^{*} \in \mathbb{C}^{*}$. Indeed,

$$
\begin{aligned}
d^{*}\left(z_{1}^{*}, z_{2}^{*}\right)=\ddot{0} & \Longleftrightarrow \sqrt{\left[\iota\left(\dot{a}_{1}-\dot{a}_{2}\right)\right]^{\ddot{ }} \ddot{+}\left(\ddot{b}_{1} \ddot{-} \ddot{b}_{2}\right)^{2}}=\ddot{0} \\
& \Longleftrightarrow \beta\left[\sqrt{\left(a_{1}-a_{2}\right)^{2}+\left(b_{1}-b_{2}\right)^{2}}\right]=\beta(0) \\
& \Longleftrightarrow\left(a_{1}-a_{2}\right)^{2}+\left(b_{1}-b_{2}\right)^{2}=0 \\
& \Longleftrightarrow a_{1}-a_{2}=0, \quad b_{1}-b_{2}=0 \\
& \Longleftrightarrow a_{1}=a_{2}, \quad b_{1}=b_{2} \\
& \Longleftrightarrow \dot{a}_{1}=\dot{a}_{2}, \quad \ddot{b}_{1}=\ddot{b}_{2} \\
& \Longleftrightarrow z_{1}^{*}=\left(\dot{a}_{1}, \ddot{b}_{1}\right)=\left(\dot{a}_{2}, \ddot{b}_{2}\right)=z_{2}^{*} .
\end{aligned}
$$


(ii) One can easily establish for all $z_{1}^{*}, z_{2}^{*} \in \mathbb{C}^{*}$ that

$$
\begin{aligned}
d^{*}\left(z_{1}^{*}, z_{2}^{*}\right) & =\sqrt{\left[\imath\left(\dot{a}_{1} \dot{-} \dot{a}_{2}\right)\right]^{2} \ddot{+}\left(\ddot{b}_{1} \ddot{-} \ddot{b}_{2}\right)^{\ddot{2}}} \\
& =\beta\left[\sqrt{\left(a_{1}-a_{2}\right)^{2}+\left(b_{1}-b_{2}\right)^{2}}\right] \\
& =\beta\left[\sqrt{\left(a_{2}-a_{1}\right)^{2}+\left(b_{2}-b_{1}\right)^{2}}\right] \\
& =d^{*}\left(z_{2}^{*}, z_{1}^{*}\right) .
\end{aligned}
$$

(iii) We show that the inequality $d^{*}\left(z_{1}^{*}, z_{2}^{*}\right) \ddot{+} d^{*}\left(z_{2}^{*}\right.$, $\left.z_{3}^{*}\right) \ddot{\geq} d^{*}\left(z_{1}^{*}, z_{3}^{*}\right)$ holds for all $z_{1}^{*}, z_{2}^{*}, z_{3}^{*} \in \mathbb{C}^{*}$. In fact,

$$
\begin{aligned}
d^{*} & \left(z_{1}^{*}, z_{2}^{*}\right) \ddot{+} d^{*}\left(z_{2}^{*}, z_{3}^{*}\right) \\
= & \sqrt{\left[\iota\left(\dot{a}_{1} \dot{-} \dot{a}_{2}\right)\right]^{2} \ddot{+}\left(\ddot{b}_{1} \ddot{-} \ddot{b}_{2}\right)^{2}} \ddot{+} \sqrt{\left[\iota\left(\dot{a}_{2}-\dot{a}_{3}\right)\right]^{2} \ddot{+}\left(\ddot{b}_{2} \ddot{-} \ddot{b}_{3}\right)^{2}} \\
= & \beta\left[\sqrt{\left(a_{1}-a_{2}\right)^{2}+\left(b_{1}-b_{2}\right)^{2}}\right] \\
& \ddot{+} \beta\left[\sqrt{\left(a_{2}-a_{3}\right)^{2}+\left(b_{2}-b_{3}\right)^{2}}\right] \\
= & \beta\left[\sqrt{\left(a_{1}-a_{2}\right)^{2}+\left(b_{1}-b_{2}\right)^{2}}+\sqrt{\left(a_{2}-a_{3}\right)^{2}+\left(b_{2}-b_{3}\right)^{2}}\right] \\
& \ddot{\beta}\left[\sqrt{\left(a_{1}-a_{3}\right)^{2}+\left(b_{1}-b_{3}\right)^{2}}\right] \\
= & \sqrt{\left[\iota\left(\dot{a}_{1} \dot{-} \dot{a}_{3}\right)\right]^{2} \ddot{+}\left(\ddot{b}_{1} \ddot{-} \ddot{b}_{3}\right)^{\ddot{2}}} \\
= & d^{*}\left(z_{1}^{*}, z_{3}^{*}\right) .
\end{aligned}
$$

Therefore, $d^{*}$ is a metric over $\mathbb{C}^{*}$.

Now, we can show that the metric space $\left(\mathbb{C}^{*}, d^{*}\right)$ is complete. Let $\left(z_{n}^{*}\right)_{n \in \mathbb{N}}$ be an arbitrary Cauchy sequence in $\mathbb{C}^{*}$. In this case, for all $\varepsilon \ddot{>0}$ there exists an $n_{0} \in \mathbb{N}$ such that $d^{*}\left(z_{m}^{*}, z_{n}^{*}\right) \ddot{<} \varepsilon$ for all $m, n \geq n_{0}$. Let $z_{m}^{*}=\left(\dot{a}_{m}, \ddot{b}_{m}\right) \in \mathbb{C}^{*}$ and $m \in \mathbb{N}$. Then,

$$
\begin{aligned}
d^{*}\left(z_{m}^{*}, z_{n}^{*}\right) & =\sqrt{\left[\iota\left(\dot{a}_{m} \dot{-} \dot{a}_{n}\right)\right]^{2} \ddot{+}\left(\ddot{b}_{m} \ddot{-} \ddot{b}_{n}\right)^{\ddot{2}}} \\
& =\beta\left[\sqrt{\left(a_{m}-a_{n}\right)^{2}+\left(b_{m}-b_{n}\right)^{2}}\right] \\
\ddot{<} \varepsilon & =\beta\left(\varepsilon^{\prime}\right) .
\end{aligned}
$$

Thus we obtain that

$$
\sqrt{\left(a_{m}-a_{n}\right)^{2}+\left(b_{m}-b_{n}\right)^{2}}<\varepsilon^{\prime} .
$$

On the other hand, since the following inequalities:

$$
\begin{gathered}
\left|a_{m}-a_{n}\right|=\sqrt{\left(a_{m}-a_{n}\right)^{2}} \leq \sqrt{\left(a_{m}-a_{n}\right)^{2}+\left(b_{m}-b_{n}\right)^{2}}, \\
\left|b_{m}-b_{n}\right|=\sqrt{\left(b_{m}-b_{n}\right)^{2}} \leq \sqrt{\left(a_{m}-a_{n}\right)^{2}+\left(b_{m}-b_{n}\right)^{2}}
\end{gathered}
$$

hold we therefore have by (32) that $\left|a_{m}-a_{n}\right|<\varepsilon^{\prime}$ and $\mid b_{m}-$ $b_{n} \mid<\varepsilon^{\prime}$. This means that $\left(a_{n}\right)$ and $\left(b_{n}\right)$ are Cauchy sequences with real terms. Since $\mathbb{R}$ is complete, it is clear that for every $\varepsilon^{\prime}>0$ there exists an $n_{1} \in \mathbb{N}$ such that $\left|a_{n}-a\right|<\varepsilon^{\prime} / 2$ for all $n \geq n_{1}$ and for every $\varepsilon^{\prime}>0$ there exists an $n_{2} \in \mathbb{N}$ such that $\left|b_{n}-b\right|<\varepsilon^{\prime} / 2$ for all $n \geq n_{2}$.

Define, $z^{*} \in \mathbb{C}^{*}$ by $z^{*}=(\dot{a}, \ddot{b})$. Then, we have

$$
\begin{aligned}
d^{*}\left(z_{n}^{*}, z^{*}\right) & =\sqrt{\left[\iota\left(\dot{a}_{n} \dot{-} \dot{a}\right)\right]^{\ddot{2}} \ddot{+}\left[\ddot{b}_{n} \ddot{-} \ddot{b}\right]^{2}} \\
& =\beta\left[\sqrt{\left(a_{n}-a\right)^{2}+\left(b_{n}-b\right)^{2}}\right] \\
& \ddot{\leq} \beta\left[\sqrt{\left(a_{n}-a\right)^{2}}+\sqrt{\left(b_{n}-b\right)^{2}}\right] \\
& =\beta\left(\left|a_{n}-a\right|+\left|b_{n}-b\right|\right) \\
& \ddot{\leq} \beta\left(\frac{\varepsilon^{\prime}}{2}+\frac{\varepsilon^{\prime}}{2}\right) \\
& =\beta\left(\varepsilon^{\prime}\right)=\varepsilon .
\end{aligned}
$$

Hence, $\left(\mathbb{C}^{*}, d^{*}\right)$ is a complete metric space.

Since $\mathbb{C}^{*}$ is a complete metric space with the metric $d^{*}$ defined by (10) induced by the norm $\ddot{\|} \cdot \ddot{\|}$, as a direct consequence of Theorem 6 , we have the following.

Corollary 7. $\mathbb{C}^{*}$ is a Banach space with the norm $\ddot{\|} \cdot \ddot{\|}$ defined by

$$
\ddot{\|} z^{*} \ddot{\|}=\sqrt{[\iota(\dot{a})]^{\ddot{2}} \ddot{+}(\ddot{b})^{\ddot{2}}} ; \quad z^{*}=(\dot{a}, \ddot{b}) \in \mathbb{C}^{*} .
$$

\section{Sequence Spaces over Non-Newtonian Complex Field}

In this section, we define the sets $\omega^{*}, \ell_{\infty}^{*}, c^{*}, c_{0}^{*}$, and $\ell_{p}^{*}$ of all, bounded, convergent, null, and absolutely $p$-summable sequences over the non-Newtonian complex field $\mathbb{C}^{*}$ which correspond to the sets $\omega, \ell_{\infty}, c, c_{0}$, and $\ell_{p}$ over the complex field $\mathbb{C}$, respectively. That is to say that

$$
\begin{aligned}
& \omega^{*}:=\left\{z^{*}=\left(z_{k}^{*}\right): z_{k}^{*} \in \mathbb{C}^{*} \forall k \in \mathbb{N}\right\}, \\
& \ell_{\infty}^{*}:=\left\{z^{*}=\left(z_{k}^{*}\right) \in \omega^{*}: \sup _{k \in \mathbb{N}} \ddot{\|} z_{k}^{*} \ddot{\|} \ddot{<} \infty\right\} \text {, } \\
& c^{*}:=\left\{z^{*}=\left(z_{k}^{*}\right) \in \omega^{*}: \exists l^{*} \in \mathbb{C}^{*} \ni \lim _{k \rightarrow \infty}^{*} z_{k}^{*}=l^{*}\right\}, \\
& c_{0}^{*}:=\left\{z^{*}=\left(z_{k}^{*}\right) \in \omega^{*}: \lim _{k \rightarrow \infty}^{*} z_{k}^{*}=\theta^{*}\right\} \text {, }
\end{aligned}
$$

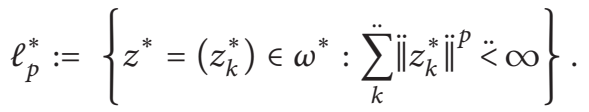

For simplicity in notation, here and in what follows, the summation without limits runs from 0 to $\infty$. One can easily 
see that the set $\omega^{*}$ forms a vector space over $\mathbb{C}^{*}$ with respect to the algebraic operations addition $(+)$ and scalar multiplication $(\times)$ defined on $\omega^{*}$, as follows:

$$
\begin{aligned}
+: \omega^{*} \times \omega^{*} & \longrightarrow \omega^{*} \\
\left(z^{*}, t^{*}\right) & \longmapsto z^{*}+t^{*}=\left(z_{k}^{*} \oplus t_{k}^{*}\right) ; \\
z^{*} & =\left(z_{k}^{*}\right), t^{*}=\left(t_{k}^{*}\right) \in \omega^{*}, \\
\times: \mathbb{C}^{*} \times \omega^{*} & \longrightarrow \omega^{*} \\
\left(\alpha, z^{*}\right) & \longmapsto \alpha \times z^{*}=\left(\alpha \odot z_{k}^{*}\right) ; \\
z^{*} & =\left(z_{k}^{*}\right) \in \omega^{*}, \alpha \in \mathbb{C}^{*} .
\end{aligned}
$$

Theorem 8. Define the function $d_{\omega^{*}}$ by

$$
\begin{aligned}
& d_{\omega^{*}}: \omega^{*} \times \omega^{*} \longrightarrow B^{\prime} \subseteq B \\
& \left(z^{*}, t^{*}\right) \longmapsto d_{\omega^{*}}\left(z^{*}, t^{*}\right) \\
& \quad=\sum_{k} \mu_{k} \ddot{\times}\left\{d^{*}\left(z_{k}^{*}, t_{k}^{*}\right) \ddot{l}\left[\ddot{1} \ddot{+} d^{*}\left(z_{k}^{*}, t_{k}^{*}\right)\right]\right\},
\end{aligned}
$$

where $\left(\mu_{k}\right) \in B^{\prime} \subseteq B$ such that $\ddot{\sum}_{k} \mu_{k}$ is convergent with $\mu_{k} \ddot{>} \ddot{0}$ for all $k \in \mathbb{N}$. Then, $\left(\omega^{*}, d_{\omega^{*}}\right)$ is a metric space.

Proof. We show that $d_{\omega^{*}}$ satisfies the metric axioms on the space $\omega^{*}$ of all non-Newtonian complex valued sequences.

(i) First we show that $d_{\omega^{*}}\left(z^{*}, t^{*}\right) \ddot{\geq} 0$ for all $z^{*}, t^{*} \in \omega^{*}$.

Because $\left(\mathbb{C}^{*}, d^{*}\right)$ is a metric space, we have $d^{*}\left(z_{k}^{*}, t_{k}^{*}\right) \ddot{\geq} 0$; $z_{k}^{*}, t_{k}^{*} \in \mathbb{C}^{*}$ and $\ddot{\mathrm{I}} \ddot{+} d^{*}\left(z_{k}^{*}, t_{k}^{*}\right) \ddot{\geq}$. Hence, we obtain that

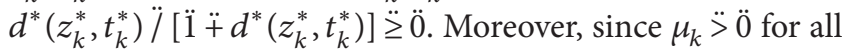
$k \in \mathbb{N}$, we have

$$
\mu_{k} \ddot{x}\left\{d^{*}\left(z_{k}^{*}, t_{k}^{*}\right) \ddot{l}\left[\ddot{1} \ddot{+} d^{*}\left(z_{k}^{*}, t_{k}^{*}\right)\right]\right\} \ddot{\geq} 0 .
$$

This means that

$$
d_{\omega^{*}}\left(z^{*}, t^{*}\right)=\sum_{k} \mu_{k} \ddot{x}\left\{d^{*}\left(z_{k}^{*}, t_{k}^{*}\right) \ddot{l}\left[\ddot{1} \ddot{+} d^{*}\left(z_{k}^{*}, t_{k}^{*}\right)\right]\right\} \ddot{\geq} \ddot{0} .
$$

(ii) We show that $d_{\omega^{*}}\left(z^{*}, t^{*}\right)=\ddot{0}$ iff $z^{*}=t^{*}$. In this situation, one can see that

$$
\begin{array}{r}
d_{\omega^{*}}\left(z^{*}, t^{*}\right) \\
=\sum_{k} \mu_{k} \ddot{x}\left\{d^{*}\left(z_{k}^{*}, t_{k}^{*}\right) \ddot{l}\left[\ddot{1} \ddot{+} d^{*}\left(z_{k}^{*}, t_{k}^{*}\right)\right]\right\}=\ddot{0} \\
\Longleftrightarrow \mu_{k} \ddot{x}\left\{d^{*}\left(z_{k}^{*}, t_{k}^{*}\right) \ddot{l}\left[\ddot{1} \ddot{+} d^{*}\left(z_{k}^{*}, t_{k}^{*}\right)\right]\right\}=\ddot{0}, \\
\forall k \in \mathbb{N}
\end{array}
$$

$$
\begin{aligned}
& \Longleftrightarrow d^{*}\left(z_{k}^{*}, t_{k}^{*}\right) \ddot{/}\left[\ddot{1} \ddot{+} d^{*}\left(z_{k}^{*}, t_{k}^{*}\right)\right]=\ddot{0},
\end{aligned}
$$

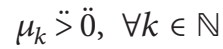

$$
\begin{aligned}
& \Longleftrightarrow d^{*}\left(z_{k}^{*}, t_{k}^{*}\right)=\ddot{0} ; \quad d^{*}\left(z_{k}^{*}, t_{k}^{*}\right) \ddot{\geq} 0 \ddot{0}, \forall k \in \mathbb{N} \\
& \Longleftrightarrow z_{k}^{*}=t_{k}^{*} ; \quad d^{*} \text { metric, } \forall k \in \mathbb{N} \\
& \Longleftrightarrow z^{*}=\left(z_{k}^{*}\right)=\left(t_{k}^{*}\right)=t^{*} \text {. }
\end{aligned}
$$

(iii) We show that $d_{\omega^{*}}\left(z^{*}, t^{*}\right)=d_{\omega^{*}}\left(t^{*}, z^{*}\right)$ for $z^{*}=$ $\left(z_{k}^{*}\right), t^{*}=\left(t_{k}^{*}\right) \in \omega^{*}$. First, we know that $d^{*}$ is a metric over $\mathbb{C}^{*}$. Thus,

$$
\begin{aligned}
d_{\omega^{*}}\left(z^{*}, t^{*}\right) & =\sum_{k} \mu_{k} \ddot{x}\left\{d^{*}\left(z_{k}^{*}, t_{k}^{*}\right) \ddot{l}\left[\ddot{1} \ddot{+} d^{*}\left(z_{k}^{*}, t_{k}^{*}\right)\right]\right\} \\
& =\sum_{k} \mu_{k} \ddot{x}\left\{d^{*}\left(t_{k}^{*}, z_{k}^{*}\right) \ddot{l}\left[\ddot{1} \ddot{+} d^{*}\left(t_{k}^{*}, z_{k}^{*}\right)\right]\right\} \\
& =d_{\omega^{*}}\left(t^{*}, z^{*}\right) .
\end{aligned}
$$

(iv) We show that $d_{\omega^{*}}\left(z^{*}, t^{*}\right) \ddot{+} d_{\omega^{*}}\left(t^{*}, u^{*}\right) \ddot{\geq} d_{\omega^{*}}\left(z^{*}, u^{*}\right)$ holds for $z^{*}=\left(z_{k}^{*}\right), t^{*}=\left(t_{k}^{*}\right), u^{*}=\left(u_{k}^{*}\right) \in \omega^{*}$. Again, using the fact that $\left(\mathbb{C}^{*}, d^{*}\right)$ is a metric space, it is easy to see by Lemma 4 that

$$
\begin{aligned}
& d_{\omega^{*}}\left(z^{*}, u^{*}\right) \\
& =\sum_{k} \mu_{k} \ddot{x}\left\{d^{*}\left(z_{k}^{*}, u_{k}^{*}\right) \ddot{l}\left[\ddot{1} \ddot{+} d^{*}\left(z_{k}^{*}, u_{k}^{*}\right)\right]\right\} \\
& \ddot{\leq} \sum_{k} \mu_{k} \ddot{x}\left\{\left[d^{*}\left(z_{k}^{*}, t_{k}^{*}\right) \ddot{+} d^{*}\left(t_{k}^{*}, u_{k}^{*}\right)\right]\right. \\
& \left.\ddot{j}\left\{\ddot{1} \ddot{+}\left[d^{*}\left(z_{k}^{*}, t_{k}^{*}\right) \ddot{+} d^{*}\left(t_{k}^{*}, u_{k}^{*}\right)\right]\right\}\right\} \\
& \ddot{\leq} \sum_{k} \mu_{k} \ddot{x}\left\{\left\{d^{*}\left(z_{k}^{*}, t_{k}^{*}\right) \ddot{l}\left[\ddot{1} \ddot{+} d^{*}\left(z_{k}^{*}, t_{k}^{*}\right)\right]\right\}\right. \\
& \left.\ddot{+}\left\{d^{*}\left(t_{k}^{*}, u_{k}^{*}\right) \ddot{l}\left[\ddot{1} \ddot{+} d^{*}\left(t_{k}^{*}, u_{k}^{*}\right)\right]\right\}\right\} \\
& =\sum_{k} \mu_{k} \ddot{x}\left\{d^{*}\left(z_{k}^{*}, t_{k}^{*}\right) \ddot{l}\left[\ddot{1} \ddot{+} d^{*}\left(z_{k}^{*}, t_{k}^{*}\right)\right]\right\} \\
& \ddot{+} \sum_{k} \mu_{k} \ddot{x}\left\{d^{*}\left(t_{k}^{*}, u_{k}^{*}\right) \ddot{l}\left[\ddot{1} \ddot{+} d^{*}\left(t_{k}^{*}, u_{k}^{*}\right)\right]\right\} \\
& =d_{\omega^{*}}\left(z^{*}, t^{*}\right) \ddot{+} d_{\omega^{*}}\left(t^{*}, u^{*}\right),
\end{aligned}
$$

as required.

Theorem 9. The set $\ell_{\infty}^{*}$ is a sequence space.

Proof. It is trivial that the inclusion $\ell_{\infty}^{*} \subset \omega^{*}$ holds.

(i) We show that $z^{*}+t^{*} \in \ell_{\infty}^{*}$ for $z^{*}=\left(z_{k}^{*}\right), t^{*}=\left(t_{k}^{*}\right) \in$ $\ell_{\infty}^{*}$. Indeed, combining the hypothesis

$$
\sup _{k \in \mathbb{N}} \ddot{\|} z_{k}^{*} \ddot{\|} \ddot{<} \infty, \quad \sup _{k \in \mathbb{N}} \ddot{\|} t_{k}^{*} \ddot{\|} \ddot{<} \infty
$$


with the fact $\ddot{\|} z_{k}^{*} \oplus t_{k}^{*} \ddot{\|} \ddot{\leq} \ddot{\|} z_{k}^{*} \ddot{\|} \ddot{+} \ddot{\|} t_{k}^{*} \ddot{\|}$ obtained from Lemma 2, we can easily derive that

$$
\sup _{k \in \mathbb{N}} \ddot{\|} z_{k}^{*} \oplus t_{k}^{*} \ddot{\|} \ddot{\leq} \sup _{k \in \mathbb{N}} \ddot{\|} z_{k}^{*} \ddot{\|} \ddot{+} \sup _{k \in \mathbb{N}} \ddot{\|} t_{k}^{*} \ddot{\|} \ddot{<} \infty .
$$

Hence, $z^{*}+t^{*} \in \ell_{\infty}^{*}$.

(ii) We show that $\alpha \times z^{*} \in \ell_{\infty}^{*}$ for any $\alpha \in \mathbb{C}^{*}$ and for $z^{*}=\left(z_{k}^{*}\right) \in \ell_{\infty}^{*}$.

Since $\ddot{\|} \alpha \odot z_{k}^{*} \ddot{\|}=\ddot{\|} \alpha \ddot{\|} \ddot{x} \ddot{\|} z_{k}^{*} \| \ddot{b}$ by Lemma 3 and $\sup _{k \in \mathbb{N}} \ddot{\|} z_{k}^{*} \| \ddot{<}$ $\infty$, it is immediate that

$$
\sup _{k \in \mathbb{N}} \ddot{\|} \alpha \odot z_{k}^{*} \ddot{\|}=\ddot{\|} \alpha \ddot{\|} \ddot{x} \sup _{k \in \mathbb{N}} \ddot{\|} z_{k}^{*} \ddot{\|} \ddot{<} \infty
$$

which means that $\alpha \times z^{*} \in \ell_{\infty}^{*}$.

Therefore, we have proved that $\ell_{\infty}^{*}$ is a subspace of the space $\omega^{*}$.

Theorem 10. Define the relation $d_{\infty}^{*}$ by

$$
\begin{aligned}
d_{\infty}^{*}: \ell_{\infty}^{*} \times \ell_{\infty}^{*} & \longrightarrow B^{\prime} \subseteq B \\
\left(z^{*}, t^{*}\right) & \longmapsto d_{\infty}^{*}\left(z^{*}, t^{*}\right)=\sup _{k \in \mathbb{N}} \ddot{\|} z_{k}^{*} \ominus t_{k}^{*} \ddot{\|} .
\end{aligned}
$$

Then, $\left(\ell_{\infty}^{*}, d_{\infty}^{*}\right)$ is a complete metric space.

Proof. One can easily show by a routine verification that $d_{\infty}^{*}$ satisfies the metric axioms on the space $\ell_{\infty}^{*}$. So, we omit the details.

Now, we prove the second part of the theorem. Let $\left(z_{m}^{*}\right)$ be a Cauchy sequence in $\ell_{\infty}^{*}$, where $z_{m}^{*}=\left(z_{k}^{* m}\right)_{k \in \mathbb{N}}$. Then, there exists a positive integer $k_{0}$ such that $d_{\infty}^{*}\left(z_{m}^{*}, z_{r}^{*}\right)=$ $\sup _{k \in \mathbb{N}} \| \ddot{l} z_{k}^{* m} \ominus z_{k}^{* r} \ddot{\|} \ddot{<} \varepsilon$ for all $m, r \in \mathbb{N}$ with $m, r>k_{0}$. For any fixed $k$, if $m, r>k_{0}$ then

$$
\ddot{\|} z_{k}^{* m} \ominus z_{k}^{* r} \ddot{\|} \ddot{<} \varepsilon
$$

In this case for any fixed $k,\left(z_{k}^{* 0}, z_{k}^{* 1}, \ldots\right)$ is a Cauchy sequence of non-Newtonian complex numbers and since $\mathbb{C}^{*}$ is complete, it converges to a $z_{k}^{*} \in \mathbb{C}^{*}$. Define $z^{*}=$ $\left(z_{0}^{*}, z_{1}^{*}, \ldots\right)$ with infinitely many limits $z_{0}^{*}, z_{1}^{*}, \ldots$ Let us show $z^{*} \in \ell_{\infty}^{*}$ and $z_{m}^{*} \rightarrow z^{*}$, as $m \rightarrow \infty$. Indeed, by (48), by letting $r \rightarrow \infty$, for $m>k_{0}$ we obtain that

$$
\ddot{\|} z_{k}^{* m} \ominus z_{k}^{*} \ddot{\|} \ddot{\leq} \varepsilon
$$

On the other hand, since $z_{m}^{*}=\left(z_{k}^{* m}\right)_{k \in \mathbb{N}} \in \ell_{\infty}^{*}$, there exists $t_{m} \in B \subseteq \mathbb{R}$ such that $\ddot{\|} z_{k}^{* m} \ddot{\|} \ddot{\leq} t_{m}$ for all $k \in \mathbb{N}$. Hence, by triangle inequality (12), the inequality

$$
\ddot{\|} z_{k}^{*} \ddot{\|} \ddot{\leq} \ddot{\|} z_{k}^{*} \ominus z_{k}^{* m} \ddot{\|} \ddot{+} \ddot{\|} z_{k}^{* m} \ddot{\|} \ddot{\leq} \varepsilon \ddot{+} t_{m}
$$

holds for all $k \in \mathbb{N}$ which is independent of $k$. Hence, $z^{*}=$ $\left(z_{k}^{*}\right)_{k \in \mathbb{N}} \in \ell_{\infty}^{*}$. By $(49)$, since $m>k_{0}$, we obtain $d_{\infty}^{*}\left(z_{m}^{*}, z^{*}\right)=$

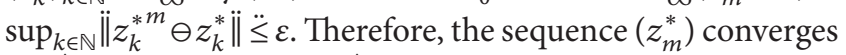
to $z^{*}$ which means that $\ell_{\infty}^{*}$ is complete.
Since it is known by Theorem 10 that $\ell_{\infty}$ is a complete metric space with the metric $d_{\infty}^{*}$ induced by the norm $\ddot{\|} \cdot \ddot{\|}_{\infty}$, defined by

$$
\ddot{\|} z^{*} \ddot{\|}_{\infty}=\sup _{k \in \mathbb{N}} \ddot{z} z_{k}^{*} \ddot{\|} ; \quad z^{*}=\left(z_{k}^{*}\right) \in \ell_{\infty}^{*},
$$

we have the following.

Corollary 11. $\ell_{\infty}^{*}$ is a Banach space with the norm $\ddot{\|} \cdot \ddot{\|}_{\infty}$ defined by (51).

Now, we give the following lemma required in proving the fact that $\ell_{p}^{*}$ is a sequence space in the case $0 \ddot{<} p \ddot{p} \ddot{1}$.

Lemma 12. Let $\ddot{0} \ddot{<p} p \ddot{<} \ddot{1}$. Then, the inequality $(\ddot{a} \ddot{+} \ddot{b})^{p} \ddot{<} \ddot{a}^{p}$ $\ddot{+} \ddot{b}^{p}$ holds for all $\ddot{a}, \ddot{b} \ddot{>} \ddot{0}$.

Proof. Let $\ddot{0} \ddot{<} p \ddot{<} \ddot{1}$ and $\ddot{a}, \ddot{b} \ddot{>} \ddot{0}$. Then, one can easily see that

$$
\begin{aligned}
& (\ddot{a} \ddot{+} \ddot{b})^{p}=\underbrace{(\ddot{a} \ddot{+} \ddot{b}) \ddot{\times} \cdots \ddot{x}(\ddot{a} \ddot{+} \ddot{b})}_{p \text {-times }} \\
& =\beta\{a+b\} \ddot{x} \cdots \ddot{x} \beta\{a+b\} \\
& =\beta\{\underbrace{\beta^{-1}[\beta(a+b)] \times \cdots \times \beta^{-1}[\beta(a+b)]}_{\beta^{-1}(p) \text {-times }}\} \\
& =\beta\left\{(a+b)^{\beta^{-1}(p)}\right\} \\
& \ddot{<} \beta\left\{a^{\beta^{-1}(p)}+b^{\beta^{-1}(p)}\right\} \\
& =\beta\left\{\beta^{-1}\left(\ddot{a}^{p}\right)+\beta^{-1}\left(\ddot{b}^{p}\right)\right\} \\
& =\ddot{a}^{p} \ddot{+} \ddot{b}^{p} \text {, }
\end{aligned}
$$

as desired.

Theorem 13. The sets $c^{*}, c_{0}^{*}$, and $\ell_{p}^{*}$ are sequence spaces, where $0 \ddot{<} p \ddot{<} \infty$.

Proof. It is not hard to establish by the similar way that $c_{0}^{*}$ and $\ell_{p}^{*}$ are the sequence spaces. So, to avoid the repetition of the similar statements, we consider only the set $c^{*}$.

It is obvious that the inclusion $c^{*} \subset \omega^{*}$ strictly holds.

(i) Let $z^{*}=\left(z_{k}^{*}\right), t^{*}=\left(t_{k}^{*}\right) \in c^{*}$. Then, there exist $l_{1}^{*}, l_{2}^{*} \in$ $\mathbb{C}^{*}$ such that $\lim _{k \rightarrow \infty}^{*} z_{k}^{*}=l_{1}^{*}$ and $\lim _{k \rightarrow \infty}^{*} t_{k}^{*}=l_{2}^{*}$. Thus, there exist $k_{1}, k_{2} \in \mathbb{N}$ such that

$$
\begin{array}{lll}
\forall \varepsilon \ddot{>0}, \quad \ddot{\|} z_{k}^{*} \ominus l_{1}^{*} \ddot{\|} \ddot{\leq} \varepsilon \ddot{2} \quad \forall k \geq k_{1}, \\
\forall \varepsilon \ddot{>0}, \quad \ddot{\|} t_{k}^{*} \ominus l_{2}^{*} \ddot{\|} \ddot{\leq} \varepsilon \ddot{2} \quad \forall k \geq k_{2} .
\end{array}
$$


Thus if we set $k_{0}=\max \left\{k_{1}, k_{2}\right\}$, by (53) we obtain for all $k \geq k_{0}$ that

$$
\begin{aligned}
& \ddot{\|}\left(z_{k}^{*} \oplus t_{k}^{*}\right) \ominus\left(l_{1}^{*} \oplus l_{2}^{*}\right) \ddot{\|} \\
& =\ddot{\|}\left(z_{k}^{*} \ominus l_{1}^{*}\right) \oplus\left(t_{k}^{*} \ominus l_{2}^{*}\right) \ddot{\|} \\
& \ddot{\leq} \ddot{\|} z_{k}^{*} \ominus l_{1}^{*} \ddot{\|} \ddot{+} \ddot{\|} t_{k}^{*} \ominus l_{2}^{*} \ddot{\|} \\
& \ddot{\leq} \varepsilon / \ddot{2} \ddot{+} \varepsilon \ddot{/} \ddot{2} \\
& =\varepsilon
\end{aligned}
$$

which means that

$$
\lim _{k \rightarrow \infty}^{*}\left(z_{k}^{*} \oplus t_{k}^{*}\right)=l_{1}^{*} \oplus l_{2}^{*}=\lim _{k \rightarrow \infty}^{*} z_{k}^{*} \oplus \lim _{k \rightarrow \infty}^{*} t_{k}^{*} .
$$

Therefore, $z^{*}+t^{*} \in c^{*}$.

(ii) Let $z^{*}=\left(z_{k}^{*}\right) \in c^{*}$ and $\alpha \in \mathbb{C}^{*} \backslash\left\{\theta^{*}\right\}$. Since $z^{*} \in c^{*}$ there exists an $l^{*} \in \mathbb{C}^{*}$ such that $\lim _{k \rightarrow \infty}^{*} z_{k}^{*}=l^{*}$, we have

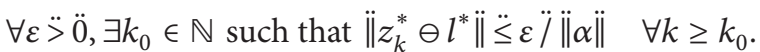

Thus, for $k \geq k_{0}$, we have

$$
\begin{aligned}
\ddot{\|}\left(\alpha \odot z_{k}^{*}\right) \ominus\left(\alpha \odot \ell^{*}\right) \ddot{\|} \\
=\ddot{\|} \alpha \odot\left(z_{k}^{*} \ominus \ell^{*}\right) \ddot{\|} \\
=\ddot{\|} \alpha \ddot{\|} \ddot{\times} \ddot{\|} z_{k}^{*} \ominus \ell^{*} \ddot{\|} \\
\ddot{\leq} \ddot{\|} \alpha \ddot{\|} \ddot{\times} \varepsilon \ddot{l} \ddot{\|} \alpha \ddot{\|} \\
=\varepsilon
\end{aligned}
$$

which implies that $\lim _{k \rightarrow \infty}^{*}\left(\alpha \odot z_{k}^{*}\right)=\alpha \odot l^{*}=\alpha \odot$ $\lim _{k \rightarrow \infty}^{*} z_{k}^{*}$ Hence, $\alpha \times z^{*} \in c^{*}$.

That is to say that $c^{*}$ is a subspace of $\omega^{*}$.

Theorem 14. $\left(c^{*}, d_{\infty}^{*}\right),\left(c_{0}^{*}, d_{\infty}^{*}\right)$, and $\left(\ell_{p}^{*}, d_{p}^{*}\right)$ are complete metric spaces, where $d_{p}^{*}$ is defined as follows:

$$
\begin{gathered}
d_{p}^{*}\left(z^{*}, t^{*}\right)= \begin{cases}\sum_{k} \ddot{\|} z_{k}^{*} \ominus t_{k}^{*} \ddot{\|}^{p}, & \ddot{0} \ddot{<} p \ddot{<} \ddot{1} \\
\left(\sum_{k} \ddot{\|} z_{k}^{*} \ominus t_{k}^{*} \ddot{\|}^{p}\right)^{1 / p}, & p \ddot{z} \ddot{1} ;\end{cases} \\
z^{*}=\left(z_{k}^{*}\right), \quad t^{*}=\left(t_{k}^{*}\right) \in \ell_{p}^{*} .
\end{gathered}
$$

Proof. We consider only the space $\ell_{p}^{*}$ with $p \ddot{\geq}$ i.

(i) For $z^{*}=\left(z_{k}^{*}\right), t^{*}=\left(t_{k}^{*}\right) \in \ell_{p}^{*}$, we establish that the two sided implication $d_{p}^{*}\left(z^{*}, t^{*}\right)=\ddot{0} \Leftrightarrow z^{*}=t^{*}$ holds. In fact,

$$
\begin{aligned}
d_{p}^{*}\left(z^{*}, t^{*}\right) & =\left(\sum_{k} \ddot{\|} z_{k}^{*} \ominus t_{k}^{*} \ddot{\|}^{p}\right)^{1 / p}=\ddot{0} \\
& \Longleftrightarrow \sum_{k} \ddot{\|} z_{k}^{*} \ominus t_{k}^{*} \ddot{\|}^{p}=\ddot{0} \\
& \Longleftrightarrow \forall k \in \mathbb{N}, \quad \ddot{\|} z_{k}^{*} \ominus t_{k}^{*} \ddot{\|}^{p}=\ddot{0}
\end{aligned}
$$

$$
\begin{aligned}
& \Longleftrightarrow \forall k \in \mathbb{N}, \quad \ddot{\|} z_{k}^{*} \ominus t_{k}^{*} \ddot{\|}=\ddot{0} \\
& \Longleftrightarrow \forall k \in \mathbb{N}, \quad z_{k}^{*}=t_{k}^{*} \\
& \Longleftrightarrow z^{*}=\left(z_{k}^{*}\right)=\left(t_{k}^{*}\right)=t^{*} .
\end{aligned}
$$

(ii) For $z^{*}=\left(z_{k}^{*}\right), t^{*}=\left(t_{k}^{*}\right) \in \ell_{p}^{*}$, we show that $d_{p}^{*}\left(z^{*}, t^{*}\right)=d_{p}^{*}\left(t^{*}, z^{*}\right)$. In this situation, since $\ddot{\|} z_{k}^{*} \ominus t_{k}^{*} \ddot{\|}=$ $\ddot{\|} t_{k}^{*} \ominus z_{k}^{*} \ddot{i}$ holds for every fixed $k \in \mathbb{N}$ it is immediate that

$$
\begin{aligned}
d_{p}^{*}\left(z^{*}, t^{*}\right) & =\left(\sum_{k} \ddot{\|} z_{k}^{*} \ominus t_{k}^{*} \ddot{\|}^{p}\right)^{1 / p} \\
& =\left(\sum_{k} \ddot{\|} t_{k}^{*} \ominus z_{k}^{*} \ddot{\|}^{p}\right)^{1 / p} \\
& =d_{p}^{*}\left(t^{*}, z^{*}\right) .
\end{aligned}
$$

(iii) By Minkowski inequality in Lemma 5, we have for $z^{*}=\left(z_{k}^{*}\right), t^{*}=\left(t_{k}^{*}\right), u^{*}=\left(u_{k}^{*}\right) \in \ell_{p}^{*}$ that

$$
\begin{aligned}
d_{p}^{*}\left(z^{*}, t^{*}\right) & =\left(\sum_{k} \ddot{\|} z_{k}^{*} \ominus t_{k}^{*} \ddot{\|}^{p}\right)^{1 / p} \\
& =\left[\sum_{k} \ddot{\|}\left(z_{k}^{*} \ominus u_{k}^{*}\right) \oplus\left(u_{k}^{*} \ominus t_{k}^{*}\right) \ddot{\|}^{p}\right]^{1 / p} \\
& \ddot{\leq}\left(\sum_{k} \ddot{\|} z_{k}^{*} \ominus u_{k}^{*} \ddot{\|}^{p}\right)^{1 / p} \ddot{+}\left(\sum_{k} \ddot{\|} u_{k}^{*} \ominus t_{k}^{*} \ddot{\|}^{p}\right)^{1 / p} \\
& =d_{p}^{*}\left(z^{*}, u^{*}\right) \ddot{+} d_{p}^{*}\left(u^{*}, t^{*}\right) .
\end{aligned}
$$

Hence, triangle inequality is satisfied by $d_{p}^{*}$ on the space $\ell_{p}^{*}$. Therefore, the function $d_{p}^{*}$ is a metric over the space $\ell_{p}^{*}$.

Now we show that the metric space $\left(\ell_{p}^{*}, d_{p}^{*}\right)$ is complete. Let $\left(z_{m}^{*}\right)_{m \in \mathbb{N}}$ be an arbitrary Cauchy sequence in the space $\ell_{p}^{*}$, where $z_{m}^{*}=\left(z_{1}^{* m}, z_{2}^{* m}, \ldots\right)$. Then, for any $\varepsilon \ddot{>} 0 \ddot{t}$ there exists an $n_{0} \in \mathbb{N}$ such that

$$
d_{p}^{*}\left(z_{m}^{*}, z_{n}^{*}\right)=\left(\sum_{k} \ddot{\|} z_{k}^{* m} \ominus z_{k}^{* n} \ddot{\|^{p}}\right)^{1 / p} \ddot{<} \varepsilon
$$

for all $m, n \geq n_{0}$. Hence, for $m, n \geq n_{0}$ and every fixed $k \in \mathbb{N}$, we obtain

$$
\ddot{\|} z_{k}^{* m} \ominus z_{k}^{* n} \ddot{\|} \ddot{<} \varepsilon
$$

If we set $k$ fixed then, it follows by (63) that $\left(z_{k}^{* 0}, z_{k}^{* 1}, \ldots\right)$ is a Cauchy sequence. Since $\mathbb{C}^{*}$ is complete, this sequence converges to a point say $z_{k}^{*}$. Let us define the sequence $z^{*}=$ $\left(z_{0}^{*}, z_{1}^{*}, \ldots\right)$ with these limits and show $z^{*} \in \ell_{p}^{*}$ and $z_{m}^{*} \rightarrow z^{*}$, 
as $m \rightarrow \infty$. Indeed, by (62), we obtain the inequality for all $m, n \in \mathbb{N}$ with $m, n \geq n_{0}$ that

$$
\sum_{k=0}^{j} \ddot{\|} z_{k}^{* m} \ominus z_{k}^{* n} \ddot{\|}^{p} \ddot{<} \varepsilon^{p} \quad \forall j \in \mathbb{N}
$$

and thus we have by letting $n \rightarrow \infty$ and $m>n_{0}$ that

$$
\sum_{k=0}^{j} \ddot{\|} z_{k}^{* m} \ominus z_{k}^{*} \ddot{\|}^{p} \ddot{<} \varepsilon^{p} \quad \forall j \in \mathbb{N}
$$

which gives as $j \rightarrow \infty$ and for all $m>n_{0}$ that

$$
\left[d_{p}^{*}\left(z_{m}^{*}, z^{*}\right)\right]^{p}=\sum_{k} \ddot{\|} z_{k}^{* m} \ominus z_{k}^{*} \ddot{\|}^{p} \ddot{<} \varepsilon^{p} .
$$

Setting $z_{k}^{*}=z_{k}^{* m} \oplus\left(z_{k}^{*} \ominus z_{k}^{* m}\right)$ and applying Lemma 5 we obtain by (66) and the fact $\left(z_{k}^{* m}\right) \in \ell_{p}^{*}$ that

$$
\begin{aligned}
\left(\sum_{k} \ddot{\|} z_{k}^{*} \ddot{\|}^{p}\right)^{1 \ddot{p}}= & \left(\sum_{k} \ddot{\|} z_{k}^{* m} \oplus\left(z_{k}^{*} \ominus z_{k}^{* m}\right) \ddot{\|}^{p}\right)^{1 / p} \\
& \ddot{\leq}\left(\sum_{k} \ddot{\|} z_{k}^{* m} \ddot{\|}^{p}\right)^{1 / p} \\
& \ddot{+}\left(\sum_{k} \ddot{\|} z_{k}^{*} \ominus z_{k}^{* m} \ddot{\|}^{p}\right)^{1 / p} \ddot{<} \infty
\end{aligned}
$$

which means that $z^{*}=\left(z_{k}^{*}\right) \in \ell_{p}^{*}$. Therefore, we see from (66) that $z_{m}^{*} \rightarrow z^{*}$. Since the arbitrary Cauchy sequence $\left(z_{m}^{*}\right)=$ $\left(z_{k}^{* m}\right)_{k, m \in \mathbb{N}} \in \ell_{p}^{*}$ is convergent, the space $\ell_{p}^{*}$ is complete.

Corollary 15. $c^{*}$ and $c_{0}^{*}$ are the Banach spaces equipped with the norm $\ddot{\|} \cdot \ddot{\|}_{\infty}$ defined in (51).

Since it is known by Theorem 14 that $\ell_{p}^{*}$ is a complete metric space with the metric $d_{p}^{*}$ induced in the case $p \ddot{\geq} \ddot{1}$ by the norm $\ddot{\|} \cdot \ddot{\|}_{p}$ and in the case $\ddot{0} \ddot{<} p \ddot{<} \ddot{\mathrm{i}}$ by the $p$-norm $\ddot{\|} \cdot \widehat{\ddot{\|}}_{p}$, defined for $z^{*}=\left(z_{k}^{*}\right) \in \ell_{p}^{*}$ by

$$
\ddot{\|} z^{*} \ddot{\|}_{p}=\left(\sum_{k} \ddot{\|} z_{k}^{*} \ddot{\|}^{p}\right)^{1 / p} \quad \ddot{\|} z^{*} \widehat{\ddot{\|}}_{p}=\sum_{k} \ddot{\|} z_{k}^{*} \ddot{\|}^{p},
$$

we have the following.

Corollary 16. The space $\ell_{p}^{*}$ is a Banach space with the norm $\ddot{\|} \cdot \ddot{\|}_{p}$ and p-norm $\ddot{\|} \cdot \widehat{\ddot{\|}}_{p}$ defined by (68).

\section{Conclusion}

We present some important inequalities such as triangle, Minkowski, and some other inequalities in the sense of nonNewtonian complex calculus which are frequently used. We state the classical sequence spaces over the non-Newtonian complex field $\mathbb{C}^{*}$ and try to understand their structure of being non-Newtonian complex vector space. There are lots of techniques that have been developed in the sense of nonNewtonian complex calculus. If the non-Newtonian complex calculus is employed instead of the classical calculus in the formulations, then many of the complicated phenomena in physics or engineering may be analyzed more easily.

As an alternative to the classical (additive) calculus, Grossman and Katz [1] introduced some new kind of calculus named as non-Newtonian calculus, geometric calculus, anageometric calculus, and bigeometric calculus. Türkmen and Başar $[6,7]$ have recentlystudied the classical sequence spaces and related topics, in the sense of geometric calculus. Quite recently, Çakmak and Başar [8] have also worked on the same subject by using non-Newtonian calculus. In the present paper, we use the non-Newtonian complex calculus instead of non-Newtonian real calculus and geometric calculus. It is trivial that in the special cases of the generators $\alpha$ and $\beta$, the non-Newtonian complex calculus gives the special kind of the following calculus:

(i) if $\alpha=\beta=I$, the identity function, then the non-Newtonian complex calculus is reduced to the classical calculus;

(ii) if $\alpha=I$ and $\beta=\exp$, then the non-Newtonian complex calculus is reduced to the geometric calculus;

(iii) if $\alpha=\exp$ and $\beta=I$, then the non-Newtonian complex calculus is reduced to the anageometric calculus;

(iv) if $\alpha=\beta=\exp$, then the non-Newtonian complex calculus is reduced to the bigeometric calculus.

Since our results are obtained by using the non-Newtonian complex calculus, they are much more general and comprehensive than those of Türkmen and Başar $[6,7]$, Çakmak and Başar [8].

Quite recently, Talo and Başar have studied the certain sets of sequences of fuzzy numbers and introduced the classical sets $\ell_{\infty}(F), c(F), c_{0}(F)$, and $\ell_{p}(F)$ consisting of the bounded, convergent, null, and absolutely $p$-summable sequences of fuzzy numbers in [9]. Nextly, they have defined the alpha-, beta-, and gamma-duals of a set of sequences of fuzzy numbers and gave the duals of the classical sets of sequences of fuzzy numbers together with the characterization of the classes of infinite matrices of fuzzy numbers transforming one of the classical set into another one. Following Bashirov et al. [2] and Uzer [3], we give the corresponding results for multiplicative calculus to the results derived for the sets of fuzzy valued sequences in Talo and Başar [9], as a beginning. As a natural continuation of this paper, we should record from now on that it is meaningful to define the alpha-, beta-, and gamma-duals of a set of sequences over the nonNewtonian complex field $\mathbb{C}^{*}$ and to determine the duals of classical spaces $\ell_{\infty}^{*}, c^{*}, c_{0}^{*}$, and $\ell_{p}^{*}$. Further, one can obtain the similar results by using another type of calculus instead of non-Newtonian complex calculus. 


\section{Acknowledgment}

The authors are very grateful to the referees for many helpful suggestions and comments about the paper which improved the presentation and its readability.

\section{References}

[1] M. Grossman and R. Katz, Non-Newtonian Calculus, Lowell Technological Institute, 1972.

[2] A. E. Bashirov, E. M. Kurpınar, and A. Özyapıcı, "Multiplicative calculus and its applications," Journal of Mathematical Analysis and Applications, vol. 337, no. 1, pp. 36-48, 2008.

[3] A. Uzer, "Multiplicative type complex calculus as an alternative to the classical calculus," Computers \& Mathematics with Applications, vol. 60, no. 10, pp. 2725-2737, 2010.

[4] A. Bashirov and M. Riza, "On complex multiplicative differentiation," TWMS Journal of Applied and Engineering Mathematics, vol. 1, no. 1, pp. 75-85, 2011.

[5] A. E. Bashirov, E. Misırlı, Y. Tandoğdu, and A. Özyapıcı, "On modeling with multiplicative differential equations," Applied Mathematics, vol. 26, no. 4, pp. 425-438, 2011.

[6] C. Türkmen and F. Başar, "Some basic results on the sets of sequences with geometric calculus," AIP Conference Proceedings, vol. 1470, pp. 95-98, 2012.

[7] C. Türkmen and F. Başar, "Some basic results on the geometric calculus," Communications de la Faculté des Sciences de l'Université d'Ankara $A_{1}$, vol. 61, no. 2, pp. 17-34, 2012.

[8] A. F. Çakmak and F. Başar, "On the classical sequence spaces and non-Newtonian calculus," Journal of Inequalities and Applications, vol. 2012, Article ID 932734, 13 pages, 2012.

[9] Ö. Talo and F. Başar, "Determination of the duals of classical sets of sequences of fuzzy numbers and related matrix transformations," Computers \& Mathematics with Applications, vol. 58, no. 4, pp. 717-733, 2009. 


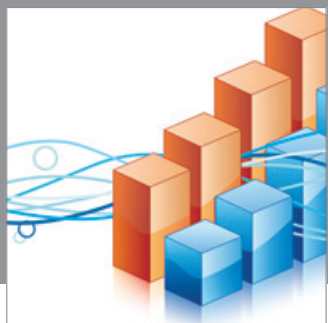

Advances in

Operations Research

mansans

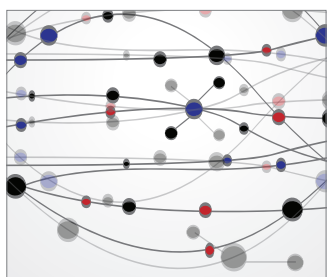

The Scientific World Journal
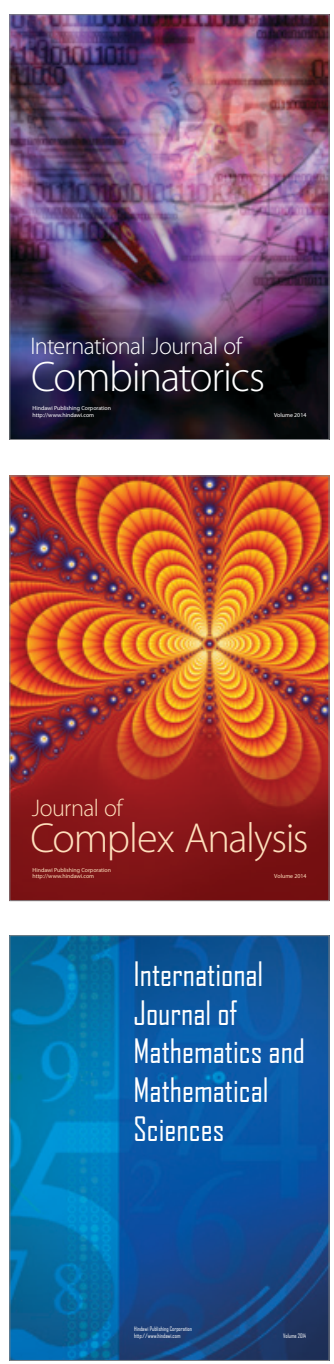
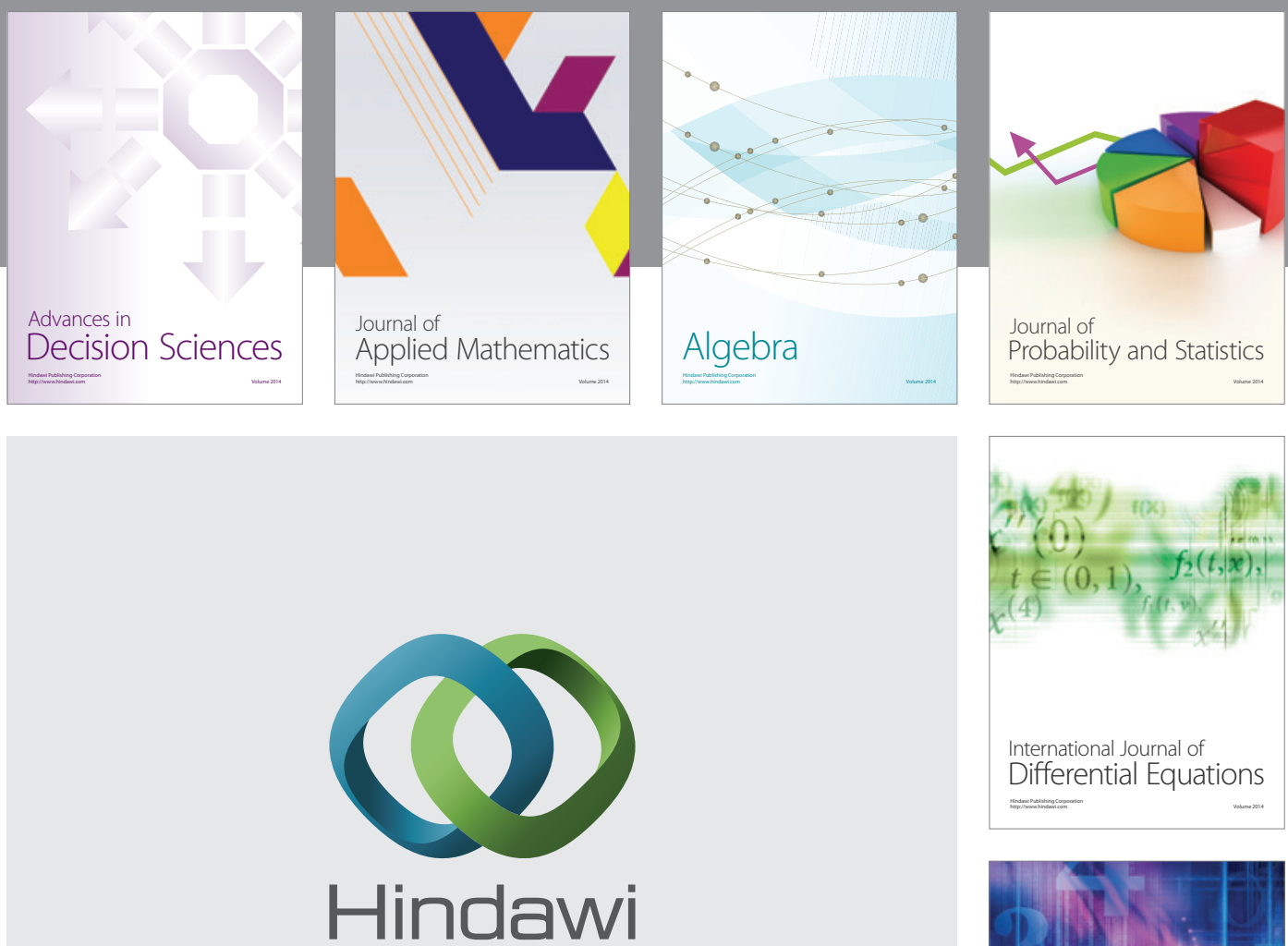

Submit your manuscripts at http://www.hindawi.com
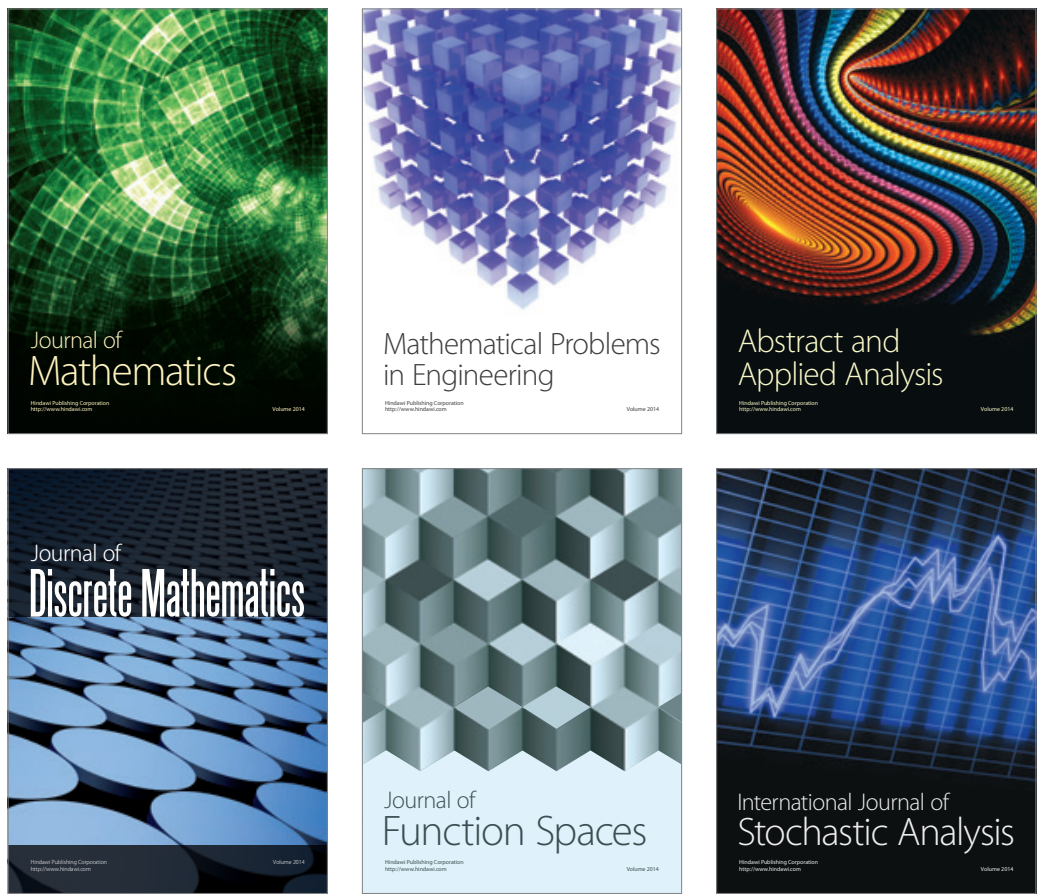

Journal of

Function Spaces

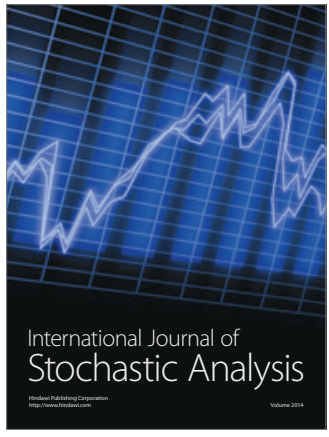

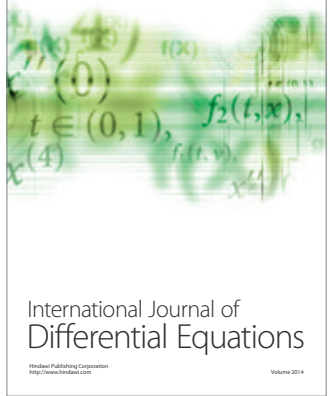
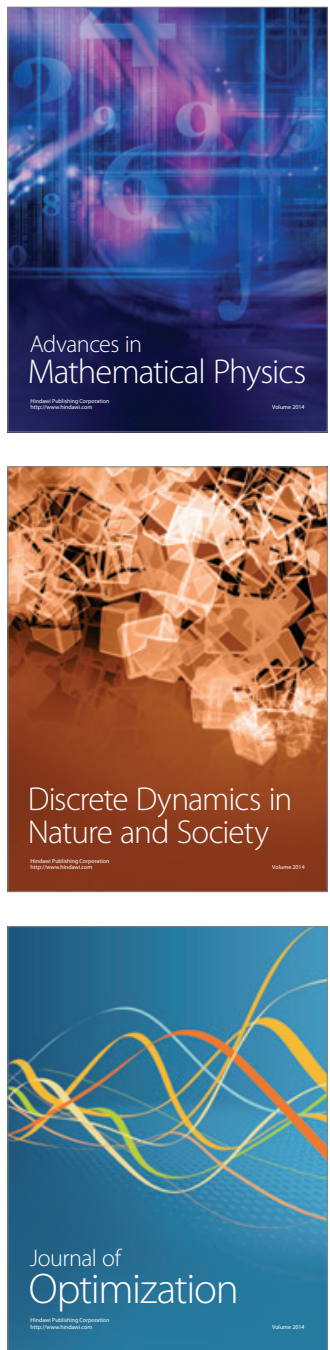